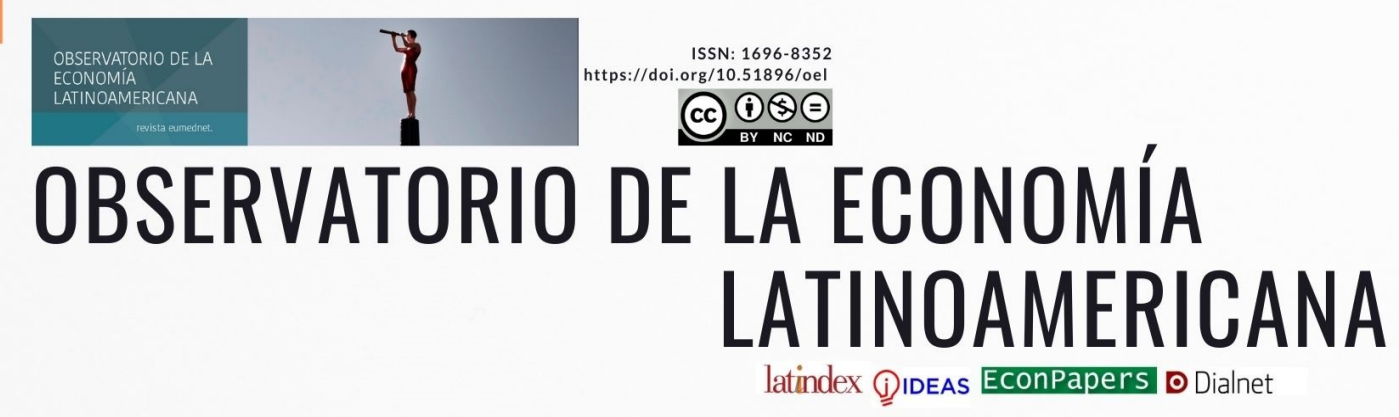

\title{
EFECTOS DEL DINERO ELECTRÓNICO BIMO EN LA RECAUDACIÓN DE TRIBUTOS DE RESTAURANTES RISE EN GUAYAQUIL
}

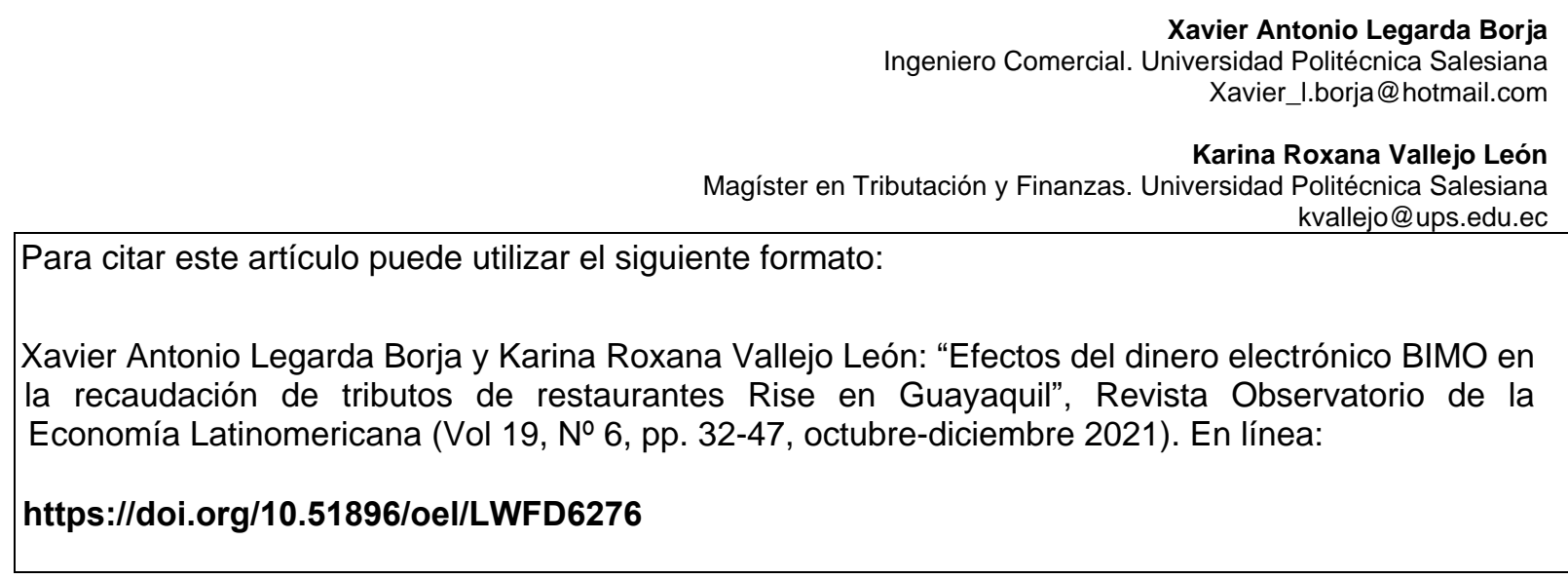

\section{RESUMEN}

El dinero electrónico BIMO se ha transformado en un instrumento para las transacciones de cobro y pago en distintos sectores de la economía ecuatoriana, sin embargo, poco se conoce sobre los efectos que genera la utilización de este medio dentro del grupo de servicio de restaurantes que pertenecen al Régimen Impositivo Simplificado, siendo un sector altamente demandante por los consumidores. Por ello, esta investigación tiene como objetivo evaluar los efectos del dinero electrónico BIMO en el nivel de recaudación de tributos en los servicios de restaurantes que pertenecen al Régimen Impositivo Simplificado mediante un estudio de correlación que permita la optimización del proceso actual de cobranza en el año 2020. La metodología utilizada tuvo un enfoque de tipo cuantitativo, correlacional explicativo, con un diseño no experimental y de corte transversal. Se recolectó los datos mediante la aplicación de la encuesta a un grupo de administradores de restaurantes que pertenecen al RISE de las parroquias Ximena, Tarqui y Rocafuerte de Guayaquil, además de entrevistas a expertos que profundizaron los contenidos. Para el análisis de correlación se utilizó el coeficiente de Pearson y el estudio del chi-cuadrado. Los resultados indicaron que el BIMO se relaciona de forma directa con la optimización del proceso de identificación y recaudación de tributos, es decir, que a mayor utilización del BIMO en las transacciones monetarias de los restaurantes, mayor será la exactitud que tendrá la información utilizada para el pago de tributos y para el acceso oportuno y rápido a trámites de recategorización de niveles RISE.

Palabras clave: BIMO, dinero electrónico, Guayaquil, Régimen Impositivo Simplificado, tributo local 


\section{IN GUAYAQUIL}

\section{ABSTRACT}

BIMO electronic money has become an instrument in the management of collection and payment transactions in different sectors of the Ecuadorian economy, however, little is known about the effects generated using this medium within the restaurant service group that belong to the Simplified Tax Regime, being a highly demanding sector by consumers. Therefore, this research aims to evaluate the effects of BIMO electronic money on the level of tax collection in restaurant services that belong to the Simplified Tax Regime through a correlation study that allows the optimization of the current collection process in the year 2020. The methodology had a quantitative, correlational explanatory, non-experimental and cross-sectional approach. The data was collected by applying the survey to a group of restaurant administrators who belong to the RISE of the Ximena, Tarqui and Rocafuerte parishes of Guayaquil, in addition to interviews with experts who deepened the contents. Pearson's coefficient and chi-square study were used for the correlation study. The results indicated that BIMO is directly related to the optimization of the process of identification and collection of taxes, that is, the greater the use of BIMO in the monetary transactions of restaurants, the greater the accuracy of the information used for the payment of taxes and for the opportune and fast access to procedures of recategorization of RISE levels.

Keywords: BIMO, electronic money, Guayaquil, level of tax collection, Simplified Tax Regime.

\section{INTRODUCCIÓN}

Este trabajo de investigación surge con la intención de conocer los efectos del dinero electrónico en el nivel de recaudación a nivel tributario en Ecuador, ya que es un tema que no ha sido explorado a profundidad, sobre todo por la cultura tributaria en el país. A partir del 2018, el Gobierno Nacional del Ecuador, junto a la Banca Privada, está fomentando el uso del sistema de Pago "Billetera Móvil" o también conocido como BIMO en las microempresas, lo que fomentaría las transacciones monetarias digitales y con ello una visión más clara del nivel de recaudación de tributos.

La utilización del dinero electrónico en Ecuador comenzó como un prototipo de pago controlado únicamente por el Banco Central, dando la oportunidad a varios sectores sociales a ser considerados en el sistema financiero privado nacional. Este sistema, al ser manejado por un Organismo Estatal, ofrecía incentivos tributarios como la reducción de hasta un dos por ciento en las declaraciones de IVA.

Sin embargo, a partir del 16 de abril del 2018, este sistema pasó a ser operado por el sistema financiero privado del Ecuador, siendo llamado Billetera Móvil o BIMO, el cual ofrece las mismas funciones que el prototipo de dinero electrónico original, aunque por el momento no abarca al $100 \%$ de cooperativas de ahorro y crédito, así como los bancos privados. Este sistema de pagos permite realizar transacciones mensuales o diarias con montos máximos de 50 dólares, los cuales pueden ser transferidos mediante el uso del dispositivo móvil. 
Para las empresas, este sistema de pago es novedoso al permitir el avance tecnológico utilizando el comercio electrónico, así como la inclusión de varios sectores al sistema financiero nacional. No obstante, existen puntos débiles en su utilización entre los cuales está la relación con el sistema tributario en el país y la falta de difusión sobre su aplicación.

Los negocios que se dedican al servicio de restaurantes y que pertenecen al Régimen Impositivo Simplificado deben tributar de acuerdo con una banda de ingresos, la cual tiene un nivel variable en distintos periodos de tiempo. Es por ello por lo que, estos negocios terminan tributando en menor o mayor medida, reflejado por los datos de la Administración Tributaria. Esto genera una ineficiencia operativa con el constante aumento de costos operativos al no reflejar valores exactos en el nivel de ingresos que genera mensualmente. Por lo tanto, muchos de estos negocios de alimentos que forman parte del Régimen Impositivo Simplificado requieren un sistema que les brinde facilidades en la actualización del nivel salarial que obtienen en sus actividades, con ello no tendrían dificultad al momento de tributar de acuerdo con su nivel de ingresos.

\section{Determinación del problema}

Para esta investigación, se ha formulado el siguiente problema: ¿Cuáles son los efectos del dinero electrónico BIMO en el nivel de recaudación de tributos en los servicios de restaurantes que pertenecen al Régimen Impositivo Simplificado en Guayaquil en el año 2020?

El objetivo general planteado para esta investigación es evaluar los efectos del dinero electrónico BIMO en el nivel de recaudación de tributos en los servicios de restaurantes que pertenecen al Régimen Impositivo Simplificado mediante un estudio de correlación que permita la optimización del proceso actual de cobranza establecido en Guayaquil en el 2020.

\section{Marco teórico referencial}

Los antecedentes teóricos del uso del dinero electrónico tanto a nivel mundial como en el Ecuador son:

Según los análisis de Reinoso, González y Gallegos (2018), la aplicación y manejo del dinero solo es representado en valores numéricos o aquellos reproducidos en billetes y monedas. Sus conclusiones principales fueron que los valores establecidos al dinero tienen su garantía monetaria equivalente al PIB o al índice de riqueza de una nación, por tanto, muestra una visión global del uso del dinero electrónico en los distintos países.

Por otro lado, Machuca, Iglesias, Ruperti y Moreira (2018) evaluaron los antecedentes del dinero electrónico a nivel mundial, el modo en que es empleado en otros países, y su funcionamiento dentro del Ecuador. Sus investigaciones demostraron que más de la mitad de los ciudadanos perciben una gran desconfianza e incertidumbre frente al uso del sistema de pago, concluyendo que la implementación de la moneda electrónica es beneficiosa debido a que al no circular el dinero físico se disminuye la importación de billetes desde los Estados Unidos y eso genera un ahorro sustancial 
para las finanzas públicas.

Así mismo, las investigaciones de Acosta, Guerra y Viteri (2018) arrojaron que, a pesar de la transformación del dinero electrónico al sistema financiero privado, aún no se ha formado un clima de confianza por parte de usuario. Sus conclusiones demuestran que existe una evolución positiva del dinero electrónico en la República del Ecuador, aunque no la esperada, por la desconfianza e incertidumbre en el sistema bancario. En tanto que, los análisis de Quiñonez, Durán, Vera y Brito (2019) explicaron que el dinero electrónico es considerado como un sistema que fortalece a la moneda en curso, y logra la inclusión de una gran cantidad de sectores al panorama fiscal. Sus conclusiones fueron que el uso del dinero electrónico por parte del Estado favorece a la población, al disminuir los gastos que proceden del uso de este servicio.

A su vez, los análisis de Cedeño, Benavides, Domo, Peña y Peña (2019) indican que la globalización en el entorno de las empresas ha llevado a buscar la satisfacción de los clientes, convirtiéndose en mercados dinámicos, concluyendo que el dinero electrónico facilita las transacciones entre usuarios y empresas, haciendo olvidar por su rapidez a medios de pago antiguos. También, según López y Baquerizo (2018), el dinero electrónico dentro del Ecuador inició con el objetivo de fortalecer la economía al ser un método incluyente, donde muchos sectores antes marginados ahora pueden acceder a la banca, por tanto, concluyeron que el sistema financiero privado está listo para ejecutar procesos de inclusión financiera buscando que la mayor cantidad de ecuatorianos accedan a servicios de billetera móvil.

\section{Bases teóricas}

\section{Generalidades del Dinero Electrónico}

El sistema de dinero electrónico, según Valencia (2019), es una opción de pago orientado a promover la inserción financiera, mejorando el sistema popular y solidario mediante el uso de líneas o dispositivos móviles que permitan el acceso a bajo costo de transacciones monetarias entre los distintos sectores productivos. A su vez, González (2019) considera que el dinero electrónico no es un dinero como tal, puesto que no está formado por efectivo o depósitos físicos, sino sus procesos se desarrollan a partir del almacenamiento en soportes electrónicos, como tarjetas físicas, virtuales, teléfonos o cualquier otro dispositivo que permita realizar procesos similares al dinero físico.

Según expresado por ambos autores, el dinero electrónico no es considerado como una moneda de valor, sino más bien se lo relaciona como un medio o sistema de pago, el cual, utilizando dispositivos digitales, puede realizar transacciones monetarias con mayor facilidad que un dinero físico.

\section{Uso del dinero electrónico en el mundo}

El dinero electrónico se ha desarrollado rápidamente a nivel global. La globalización ha planteado en gran medida el fortalecimiento de estrategias corporativas que fomenten el uso de los 
dispositivos electrónicos como vías de pago, todo por la creciente competencia empresarial sumada al comercio electrónico. En efecto, el comercio electrónico podría ser considerado como un precursor del fortalecimiento de un sistema de pago llamado dinero electrónico (Aguilar \& Meza, 2019).

Esta característica sumada a la democratización de los servicios financieros utilizando la tecnología ha logrado incluir a la mayor cantidad de personas a la banca, permitiendo el acceso a pagos, créditos y demás sin distinción de categoría social. De acuerdo con los estudios de Roa, García, Frías y Correa (2017), en Latinoamérica existen alrededor de 21 millones de cuentas bancarias electrónicas registradas, siendo los países de Brasil, República Dominicana, Colombia, Panamá, Ecuador y Perú los que más destacan en el uso del dinero electrónico.

\section{Uso de la moneda electrónico en el Ecuador}

En el Ecuador, el dinero electrónico ha tenido varios ajustes desde sus inicios. Este sistema comenzó como una moneda móvil, operada por la Banca Pública representada en el Banco Central del Ecuador, la cual se denominaba "Efectivo desde mi celular". Sin embargo, este sistema no prosperó por la falta de promoción por parte de los entes gubernamentales, lo que generó que se realice una transferencia de competencia a la banca privada (Villaroel, Ortíz, \& Proaño, 2018).

Desde ahí nace el proyecto BIMO o Billetera Móvil, el cual, de acuerdo con su página oficial web, es un servicio operado por BANRED con la cual se pueden realizar solicitudes de cobro y pago de dinero a otros usuarios que tengan la misma plataforma en sus dispositivos. Para ello, el usuario debe ingresar el valor monetario desde su dispositivo celular, escoger dentro de su lista de contactos o por medio de un código QR, y automáticamente se realizará una transferencia bancaria sin tomar en cuenta la institución privada a la que pertenezca. Esta Billetera Móvil o BIMO, se asocia directamente al número de celular y, de acuerdo con la operatividad, se asocia a la institución financiera afiliada.

Por otro lado, en el sentido tributario, hay que destacar que el proyecto anterior al BIMO, ofrecía incentivos tributarios a las personas que realicen transacciones por ese sistema. Dichos incentivos iban entre una reducción de 2 puntos porcentuales del IVA hasta el pago oportuno de las obligaciones tributarias. En este sentido, se debe destacar que aún se mantiene el pago de forma ininterrumpida, es decir, las 24 horas del día. Este aspecto es muy importante debido que las personas ya no estarían limitadas con respecto al tiempo en agencias bancarias, sino podrían hacer uso del sistema, pagando oportunamente sus obligaciones.

\section{Generalidades de la Recaudación Tributaria en el Ecuador}

En el Ecuador, existen 2 tipos de contribuyentes pasivos, los cuales son las personas naturales y las sociedades. De estas, dentro de las personas naturales se consideran dos subgrupos los cuales son los obligados a llevar contabilidad y no obligados a llevar contabilidad. Para las personas naturales existen dos tipos de regímenes con los cuales pueden realizar su declaración, el 
Régimen General RUC y el Régimen Impositivo Simplificado Ecuatoriano (SRI, 2020). Las obligaciones entre el Régimen General y el RISE varían, siendo el Régimen General más completo en cuanto a las responsabilidades con la Administración Tributaria. Las personas naturales que pertenecen al Régimen General están obligadas a emitir y entregar los respectivos comprobantes de venta que sean autorizados por el SRI y declarar y pagar los respectivos impuestos a los que estén obligados los contribuyentes.

En cambio, el RISE es un régimen voluntario, es decir, el contribuyente puede inscribirse bajo la forma simplificada siempre y cuando cumpla requisitos específicos. Esta modalidad se creó con el fin de fomentar la cultura tributaria del país y reducir los delitos fiscales (SRI, 2020).

\section{Principales problemas en el sector de restaurantes RISE a nivel tributario}

Uno de los principales inconvenientes que existe a nivel tributario es la evasión tributaria. De acuerdo con el estudio de Paredes (2016), esta puede definirse como todos los hechos que conlleven a un incumplimiento que van desde una reducción de montos obligatorios por ley a pagar al Estado Ecuatoriano hasta incrementar los egresos permitidos como deducibles por la norma impositiva vigente. Siendo el contribuyente el encargado de transmitir la información sobre el total de ingresos que percibió en un determinado periodo de tiempo, sumado a la cantidad de egresos documentados, esto da la opción de realizar una declaración no objetiva sobre los montos reales registrados, con el fin de disminuir los valores a pagar a la Administración Tributaria. Según una investigación de Quispe, Arellano, Negrete, Rodríguez y Vélez (2020) algunos de los factores de la evasión tributaria pueden ser:

- Falta de educación tributaria en la sociedad

- Escasos o nulos mecanismos estatales para el cumplimiento de la ley

- Desconocimiento de la legislación tributaria en un territorio

- Insatisfacción de los contribuyentes que no ven la retribución de sus impuestos

- Corrupción generalizada

\section{METODOLOGÍA}

\section{Diseño de investigación}

El enfoque de esta investigación es de tipo cuantitativo debido a que evaluó datos numéricos en el procesamiento de variables, así como para la recolección de datos utilizados en el análisis de la hipótesis. En cuanto al alcance de la investigación, esta es de tipo correlacional explicativa debido a que buscó comprender la interacción entre el uso del dinero electrónico BIMO y el nivel de recaudación de tributos en los servicios de restaurantes que pertenecen al Régimen Impositivo Simplificado. Además, esta investigación se diseñó de forma no experimental debido a que no se pretende controlar ni manipular las variables presentes en la investigación, únicamente se estudió el 
fenómeno en su ambiente natural para posteriormente analizar los datos recolectados. Además, es una investigación transversal debido a que se recolecta datos en un solo periodo de tiempo, siendo el año 2020 el periodo escogido para el estudio.

\section{Muestra}

La población de estudio corresponde a los negocios de comida que pertenecen al RISE de la ciudad de Guayaquil, la cual de acuerdo con datos proporcionados por la Administración Tributaria dentro de su catastro de contribuyentes activos al 2020 asciende a 1188. A partir de estos datos, se aplica un procedimiento de obtención de muestra, dado que la población de estudio es muy amplia. Por otro lado, se aplica la técnica de muestreo probabilístico por conglomerados al agrupar a los negocios de comida por parroquia urbana de la ciudad, teniendo como base las parroquias Ximena, Tarqui y Rocafuerte al ser consideradas como las más grandes de la ciudad. El procedimiento para determinar la muestra consiste en aplicar la fórmula de muestreo probabilístico, obteniendo un número considerable de negocios a los cuales se puede obtener información y dividirlo para las tres parroquias que serán objeto de estudio.

El tamaño de la muestra sería de 291 restaurantes RISE de la ciudad de Guayaquil. La hipótesis de investigación es la siguiente: Hi: El dinero electrónico BIMO optimiza el proceso de identificación y recaudación de los tributos en los servicios de restaurantes de la ciudad de Guayaquil que pertenecen al Régimen Impositivo Simplificado (RISE). Se utiliza como herramienta de recolección de datos a la encuesta, al cual será aplicada a propietarios o administradores de 291 restaurantes que pertenecen al Régimen Impositivo Simplificado en la ciudad de Guayaquil. La encuesta está formada por un cuestionario de 12 preguntas, las cuales son de tipo dicotómicas y de opción múltiple.

\section{RESULTADOS Y DISCUSIÓN}

\section{Hallazgos en las encuestas aplicadas}

Las encuestas aplicadas dieron los siguientes resultados: Del total de encuestados, el 100\% correspondiente a 291 personas indicaron que sus negocios de restaurantes pertenecen al RISE en Guayaquil. Del total de encuestados, el 55\% de estos indicaron que sí conocen o han escuchado sobre el dinero electrónico en Ecuador, correspondiendo a 160 personas encuestas, por otro lado, un $45 \%$ representado en 131 personas, indicaron que desconocen sobre lo que representa el dinero electrónico y su respectivo uso en las transacciones comerciales. De acuerdo con la pregunta 3, el $53 \%$ de los encuestados representados por 154 administradores de restaurantes RISE indicaron que sí están afiliados al dinero electrónico mientras que un 47\%, es decir, 137 administradores, indicaron que no se han afiliado a un sistema de dinero electrónico.

Los resultados de la pregunta 4, tomando como base a los 154 afiliados al sistema de dinero electrónico, indican que con un $38 \%$ perteneciente a 58 personas, estas utilizan dentro de sus 
negocios el sistema de pago de tarjetas de crédito, seguido por un $33 \%$ perteneciente a 50 personas que indican que utilizan tarjetas de débito como medio de pago. De estas, solo el $30 \%$ indica que utilizan la billetera móvil BIMO en sus negocios, es decir, 46 personas encuestadas. Según los resultados, el 58\% de los encuestados, es decir 168 personas, indican que el dinero físico es el medio de pago más utilizado en sus establecimientos. En segundo lugar, está las tarjetas de débito con un 16\%, es decir 46 personas. En tercer lugar, está las tarjetas de crédito con un 15\%, es decir 44 personas y en cuarto lugar está la billetera móvil BIMO con un $11 \%$, es decir 33 personas. Los resultados de la pregunta 6 , teniendo como base a las 46 personas que indicaron que están afiliadas al BIMO, indican que, con un $89 \%$ de los encuestados representados en 41 personas, consideran que el dinero electrónico BIMO optimiza la recaudación de ingresos en los servicios que ofrece, mientras que un $11 \%$, representado en 5 personas, considera que el BIMO no ha tenido un mayor impacto en sus operaciones.

Según los encuestados, el $51 \%$ correspondiente a 147 personas, consideran que los costos bancarios por uso del dinero electrónico si son convenientes para la economía de sus consumidores, frente a un $49 \%$, es decir 144 personas, quienes consideran que no influye estos costos en su economía. El $65 \%$ de los encuestados, es decir 189 personas, han realizado en algún momento el trámite de recategorización de RISE, frente al 35\%, es decir 102 personas, quienes no lo han realizado. Del total de encuestados, el $76 \%$ considera que el sistema de dinero electrónico BIMO favorece de forma rápida y exacta en el trámite de recategorización de RISE, siendo 221 personas con opiniones afirmativas, frente a un $24 \%$ de encuestados, es decir 70 personas, quienes consideran que el BIMO no influye en los trámites de recategorización.

Del total de encuestados, el 76\% correspondiente a 221 personas, consideran que el BIMO optimizaría la información presentada al SRI, sobre todo en el nivel de ingresos que percibe, frente a un $24 \%$ correspondiente a 70 encuestados quienes consideran que no existe mayor influencia del BIMO. Para el $60 \%$ de los encuestados, es decir 170 personas, el BIMO mejora el mecanismo de recaudación de tributos en el régimen RISE, frente a un 40\% correspondiente a 116 personas quienes consideran que el BIMO no aporta en el mecanismo de recaudación de tributos. Para los encuestados, el principal efecto del BIMO es la optimización de la información sobre los ingresos, siendo el $45 \%$ correspondiente a 132 personas. En segundo lugar, está la reducción de la evasión y elusión tributaria con un $24 \%$ correspondiente a 70 personas. En tercer lugar, está la mejora en el sistema de pago de impuestos con un $20 \%$ correspondiente a 57 personas y en cuarto lugar está la facilidad en la recategorización RISE con un 11\% correspondiente a 32 personas.

Por otro lado, utilizando los datos obtenidos en la encuesta, se utilizó la prueba Chi Cuadrado y la correlación de Pearson, para terminar si existe relación entre las variables detectadas, las cuales son el uso del dinero electrónico y la identificación y recaudación de tributos. Los resultados son los siguientes: 
Tabla 1:

Prueba Chi-cuadrado entre uso del dinero electrónico e información tributaria

Pruebas de chi-cuadrado

\begin{tabular}{l|c|c|c} 
& Valor & gl & $\begin{array}{c}\text { Significación } \\
\text { asintótica } \\
\text { (bilateral) }\end{array}$ \\
\hline $\begin{array}{l}\text { Chi-cuadrado de } \\
\text { Pearson }\end{array}$ & $63,120^{\mathrm{a}}$ & 3 & $<, 001$ \\
\hline $\begin{array}{l}\text { Razón de verosimilitud } \\
\text { Asociación lineal por }\end{array}$ & 84,623 & 3 & $<, 001$ \\
\hline lineal & 52,232 & 1 & $<, 001$ \\
\hline $\mathrm{N}$ de casos válidos & 291 & & \\
\hline
\end{tabular}

a. 0 casillas $(0,0 \%)$ han esperado un recuento menor que 5 .

El recuento mínimo esperado es 7,94 .

Elaboración: Autor (2021)

Con un Chi-cuadrado de Pearson de 63,120, gl=3 y $p=<0,001$, como la significancia es menor que 0.05 se rechaza la afirmación de que el dinero electrónico no optimiza el proceso de identificación de la información tributaria en contribuyentes RISE, validando la importancia del dinero electrónico en el proceso de recaudación de tributos.

Tabla 2:

Correlación de Pearson Uso del dinero electrónico y optimización de información

\section{Correlaciones}

\begin{tabular}{llr|r} 
& & $\begin{array}{c}\text { Uso_del_clie } \\
\text { nte_al_siste } \\
\text { ma }\end{array}$ & $\begin{array}{c}\text { BIMO_optimiz } \\
\text { a_informació } \\
\text { n_tributaria }\end{array}$ \\
\hline $\begin{array}{l}\text { Uso_del_cliente_al_siste } \\
\text { ma }\end{array}$ & Correlación de Pearson & 1 &, $424^{\mathrm{N}}$ \\
\cline { 2 - 4 } & Sig. (bilateral) & & $<, 001$ \\
\cline { 2 - 4 } & $\mathrm{N}$ & 291 & 291 \\
\hline $\begin{array}{l}\text { BIMO_optimiza_informaci } \\
\text { ón_tributaria }\end{array}$ & Correlación de Pearson &, $424^{\mathrm{N}}$ & 1 \\
\cline { 2 - 4 } & Sig. (bilateral) & $<, 001$ & \\
\cline { 2 - 4 } & $\mathrm{N}$ & 291 & 291 \\
\hline
\end{tabular}

**. La correlación es significativa en el nivel 0,01 (bilateral).

Elaboración: Autor (2021)

En la prueba de Pearson, se obtiene datos de una significancia bilateral $<0.001$, siendo menor al nivel propuesto de $\mathbf{0 . 0 1}$, por lo tanto, se valida el rechazo de la afirmación que indica que el dinero electrónico no optimiza el proceso de identificación de la información tributaria en contribuyentes RISE. Por otro lado, se encontró una asociación lineal estadísticamente significativa 
moderada y proporcional con valores de $r p=0.424$ y $p=<0.001$. En la tabla 3 , se muestra los resultados entre las variables uso del dinero electrónico y recaudación de tributos:

Tabla 3:

Prueba Chi-cuadrado uso del dinero electrónico y recaudación de tributos

\begin{tabular}{|c|c|c|c|}
\hline \multicolumn{4}{|c|}{ Pruebas de chi-cuadrado } \\
\hline & Valor & gl & $\begin{array}{c}\text { Significación } \\
\text { asintótica } \\
\text { (bilateral) }\end{array}$ \\
\hline $\begin{array}{l}\text { Chi-cuadrado de } \\
\text { Pearson }\end{array}$ & $135,615^{a}$ & 3 & $<, 001$ \\
\hline Razón de verosimilitud & 172,937 & 3 & $<, 001$ \\
\hline $\begin{array}{l}\text { Asociación lineal por } \\
\text { lineal }\end{array}$ & 110,727 & 1 & $<, 001$ \\
\hline $\mathrm{N}$ de casos válidos & 291 & & \\
\hline
\end{tabular}

Elaboración: Autor (2021)

Con un Chi-cuadrado de Pearson de 135,615, gl=3 y $p=<0,001$, como la significancia es menor que 0.05 se rechaza la afirmación de que el dinero electrónico no optimiza el proceso de recaudación de tributos en contribuyentes RISE, validando la importancia del dinero electrónico en la exactitud de la información presentada al momento de tributar de acuerdo con bandas de ingreso.

Tabla 4:

Correlación de Pearson entre uso del dinero electrónico y recaudación de tributos

\section{Correlaciones}

\begin{tabular}{llr|r} 
& & $\begin{array}{c}\text { Uso_del_clie } \\
\text { nte_al_siste } \\
\text { ma }\end{array}$ & $\begin{array}{c}\text { BlMO_optimiz } \\
\text { a_recaudació } \\
\text { n_tributos }\end{array}$ \\
\hline $\begin{array}{l}\text { Uso_del_cliente_al_siste } \\
\text { ma }\end{array}$ & Correlación de Pearson & 1 &, $618^{\star \star}$ \\
\cline { 2 - 4 } & Sig. (bilateral) & & $<, 001$ \\
\cline { 2 - 4 } & $\mathrm{N}$ & 291 & 291 \\
\hline $\begin{array}{l}\text { BIMO_optimiza_recaudac } \\
\text { ión_tributos }\end{array}$ & Correlación de Pearson &, $618^{\star \star}$ & 1 \\
\cline { 2 - 4 } & Sig. (bilateral) & $<, 001$ & \\
\cline { 2 - 4 } & $\mathrm{N}$ & 291 & 291 \\
\hline
\end{tabular}

**. La correlación es significativa en el nivel 0,01 (bilateral).

Elaboración: Autor (2021)

En la prueba de Pearson, se obtiene datos de una significancia bilateral $<0.001$, siendo menor al nivel propuesto de 0.01 , por lo tanto, se rechaza la afirmación que indica que el dinero electrónico no optimiza el proceso recaudación de tributos en contribuyentes RISE. Por otro lado, se 
encontró una asociación lineal estadísticamente significativa fuerte y proporcional con valores de $\mathrm{rp}=0.618$. A continuación, se presenta, los resultados del estadístico X2 entre las variables de uso del BIMO y efectos en el recaudo tributario:

Tabla 5:

Prueba chi-cuadrado entre uso del dinero electrónico y efectos en la recaudación

\section{Pruebas de chi-cuadrado}

\begin{tabular}{l|c|c|c} 
& Valor & gl & $\begin{array}{c}\text { Significación } \\
\text { asintótica } \\
\text { (bilateral) }\end{array}$ \\
\hline $\begin{array}{l}\text { Chi-cuadrado de } \\
\text { Pearson }\end{array}$ & $69,542^{\text {a }}$ & 9 & $<, 001$ \\
\hline $\begin{array}{l}\text { Razón de verosimilitud } \\
\text { Asociación lineal por }\end{array}$ & 90,268 & 9 & $<, 001$ \\
\hline lineal & 31,703 & 1 & $<, 001$ \\
\hline $\mathrm{N}$ de casos válidos & 291 & & \\
\hline
\end{tabular}

a. 2 casillas $(12,5 \%)$ han esperado un recuento menor que 5 .

El recuento mínimo esperado es 3,63.

Elaboración: Autor (2021)

Con un Chi-cuadrado de Pearson de 69,542, gl=9 y $p=<0,001$, como la significancia es menor que 0.05 se rechaza la afirmación de que el dinero electrónico no optimiza el proceso de identificación y recaudación de tributos en contribuyentes RISE, validando la importancia del dinero electrónico en el proceso de verificación del nivel de ingresos para tributar correctamente, reduciendo la elusión y evasión. Por otro lado, la tabla 6 expone los valores de Pearson de las variables en estudio:

Tabla 6:

Correlación de Pearson entre uso del dinero electrónico y efectos en la recaudación

\begin{tabular}{|c|c|c|c|}
\hline \multicolumn{4}{|c|}{ Correlaciones } \\
\hline & & $\begin{array}{c}\text { Uso_del_clie } \\
\text { nte_al_siste } \\
\text { ma }\end{array}$ & $\begin{array}{c}\text { Efecto_del_BI } \\
\text { MO_en_recau } \\
\text { dación_de_tri } \\
\text { butos }\end{array}$ \\
\hline \multirow{3}{*}{$\begin{array}{l}\text { Uso_del_cliente_al_siste } \\
\text { ma }\end{array}$} & Correlación de Pearson & 1 &, $331^{\star *}$ \\
\hline & Sig. (bilateral) & & $<, 001$ \\
\hline & $\mathrm{N}$ & 291 & 291 \\
\hline \multirow{3}{*}{$\begin{array}{l}\text { Efecto_del_BIMO_en_rec } \\
\text { audación_de_tributos }\end{array}$} & Correlación de Pearson &, $331^{\star x}$ & 1 \\
\hline & Sig. (bilateral) & $<, 001$ & \\
\hline & $\mathrm{N}$ & 291 & 291 \\
\hline
\end{tabular}

**. La correlación es significativa en el nivel 0,01 (bilateral).

Elaboración: Autor (2021) 


\section{Hallazgos de la aplicación de las entrevistas}

Entrevista al Analista en banca electrónica: Ing. José Solano Pulla, maestrando en Dirección Financiera

El Ing. José Solano labora como analista de banca electrónica y medios digitales en una reconocida institución financiera público-privada, que en los últimos años ha impulsado el uso del dinero electrónico BIMO y el desarrollo de aplicaciones móviles de dinero digital, fomentando la cultura financiera en el país. Los puntos más destacados de la entrevista son los siguientes:

La iniciativa del BIMO ha sido fundamental para establecer un cambio en las transacciones monetarias del Ecuador, a partir de esta herramienta, muchas personas que antes no accedían a cuentas bancarias ahora lo pueden hacer desde sus dispositivos móviles, representando un ahorro en la economía del hogar. EI BIMO como tal favorece al proceso de recaudación de tributos, puesto que los contribuyentes pueden acceder a pagos de forma remota directamente a canales virtuales con el SRI, dejando atrás las interminables filas en las instituciones bancarias, sobre todo en días muy cercano al vencimiento del pago del tributo. En el país es muy difícil el acceso a canales sobre capacitaciones en finanzas a los microempresarios, por lo que el BIMO, podría darle de forma diaria o mensual, estadísticas sobre los distintos cobros y pagos realizados, teniendo mayor control en sus finanzas.

\section{Entrevista a la Técnico en Tesorería: Ing. Génesis Herrera Mero, experta en banca virtual y medios de pago digitales}

La Ing. Génesis Herrera labora como Técnico en Tesorería en una reconocida empresa siderúrgica, además por su vínculo con la banca, es experta en transacciones en banca virtual y medios de pago digitales, aportando valiosos conocimientos sobre casos especiales de BIMO que se detallan a continuación:

El BIMO, así como otros canales digitales que ofrecen las instituciones del país para las distintas transacciones monetarias aportan en el desarrollo del sistema financiero nacional, sobre todo porque a nivel global muchos países están considerando la utilización de estos medios de pago por su versatilidad y seguridad. Aún falta mucho más por mejorar en el BIMO, puesto que, si bien es cierto, esta herramienta consolida el proceso de recaudación de tributos, no se ha generalizado su uso y la difusión de la billetera móvil es casi nula. Para el contribuyente RISE el BIMO es una excelente herramienta en la gestión de cobros y pagos, a pesar de que son personas naturales sin obligación a tener registros contables, esta herramienta favorece un mejor control al momento de tomar decisiones en los negocios o evaluar los flujos de dinero que generen. 


\section{DISCUSIÓN}

El dinero electrónico BIMO es una herramienta que se adapta a las exigencias de un mercado globalizado. Según los aportes teóricos investigados, este dinero electrónico cada vez más es utilizado en diversos países, debido a los bajos costos que presupone en su utilización. En el Ecuador, la iniciativa gubernamental logró incluir sectores económicos que antes eran excluidos por la banca privada, sobre todo porque los usuarios no cumplían requisitos mínimos para aperturar cuentas de ahorro o corriente. A nivel tributario, el prototipo de dinero electrónico original suponía un ahorro en transacciones económicas al establecer una devolución de dos puntos porcentuales en la compra de bienes y servicios.

Sin embargo, este prototipo de dinero electrónico pasó a ser administrado por el sistema financiero privado, transformándose en billetera móvil o BIMO. Su forma de operar es la misma que el prototipo original, siendo utilizado por vía celular, donde los usuarios tienen la opción de aperturar cuentas bancarias y registrar su número telefónico para realizar transacciones de cobro y pago directamente de esta plataforma. De acuerdo con las encuestas aplicadas, la mayoría de los encuestados conoce sobre el uso del dinero electrónico, sin embargo, este indicador también demuestra que falta más difusión sobre este sistema de pago, puesto que lo ideal es que esta plataforma sea utilizada al mismo nivel del dinero físico o con el uso de tarjetas magnéticas. Por otra parte, las encuestas demostraron que la mayor cantidad de personas aún prefiere el dinero físico, esto debido a que existe sectores sociales que aún son capacitados para operar dispositivos electrónicos, generando un rechazo a toda tecnología y prefiriendo lo tradicional.

A nivel económico, los encuestados manifestaron que el BIMO genera costos bajos de transacción bancaria, lo cual también es reforzado por las opiniones recogidas en la entrevista a expertos quienes consideran que existe una diferencia entre transacciones por canales electrónicos y por el uso del BIMO. Cabe destacar que, existe la creencia de que el dinero físico no genera costos, sin embargo, a nivel país, existe un gasto importante en la importación del papel moneda, teniendo que ser desembolsados casi 12 millones de dólares anuales por el mantenimiento del papel físico. A nivel fiscal, teniendo como base de estudio a los servicios de restaurantes que pertenecen al Régimen Impositivo Simplificado o RISE, este dinero electrónico favorece en la optimización de información clara y en tiempo real de los montos obtenidos por las transacciones diarias que realizan estos negocios. Sin esta herramienta, los contribuyentes RISE, al no estar obligados a mantener un registro contable, no se preocupaban de conocer el nivel de ingresos y gastos que se generaban en sus negocios.

Esto pudo comprobarse con pruebas de correlación de Pearson donde se visualizó que a mayor uso del BIMO en transacciones monetarias, mayor exactitud en la información se tendrá al momento de establecer escalas de ingreso con las cuales se tributará. Así mismo, el proceso de recaudación de tributos se realizará de forma óptima y eficiente, sobre todo porque por medio de 
sistemas electrónicos se establecerá en tiempo real el valor del tributo, sin incurrir en manipulaciones o errores humanos que generaban notas de venta con información irreal.

\section{CONCLUSIONES}

El objetivo general planteado fue "evaluar los efectos del dinero electrónico BIMO en el nivel de recaudación de tributos en los servicios de restaurantes que pertenecen al Régimen Impositivo Simplificado mediante un estudio de correlación que permita la optimización del proceso actual de cobranza establecido en la ciudad de Guayaquil en el año 2020" por lo que se puede concluir que el dinero el dinero electrónico BIMO optimiza el proceso de identificación y recaudación de los tributos en los servicios de restaurantes de la ciudad de Guayaquil que pertenecen al Régimen Impositivo Simplificado, validando la hipótesis de investigación planteada.

Por otra parte, la mayoría de los encuestados conoce el medio de pago BIMO, así mismo han implementado estas plataformas digitales a sus negocios, sin embargo, indicaron que debe existir mayor compromiso gubernamental y privado para la difusión de estas herramientas que puedan ser utilizadas por todos los ciudadanos, incluyendo sectores con cierto rechazo a la tecnología.

\section{REFERENCIAS}

Acosta, M., Guerra, A., \& Viteri, F. (5 de enero de 2018). Evolución y perspectivas del dinero electrónico en el Ecuador. Obtenido de Revista Científica Dominio de las Ciencias: https://dialnet.unirioja.es/servlet/articulo?codigo $=631325$

Aguilar, S., \& Meza, I. (2019). Transición al dinero electrónico y su impacto en la competitividad de la microempresa. Obtenido de Universidad Veracruzana: https://www.uv.mx/iic/files/2018/01/02A041153.pdf

BANRED. (2019). ¿Qué es BIMO? Obtenido de BIMO: https://bimo.ec/que-es-bimo/

Campuzano, J., Chávez, G., \& Maza, J. (24 de agosto de 2018). El fracaso del dinero electrónico en el Ecuador. Obtenido de Researchgate: https://www.researchgate.net/publication/327219691_El_fracaso_del_dinero_electronico_en_ Ecuador

Carrión, F. (14 de agosto de 2019). Resolución 534-2019. Obtenido de Junta Monetaria Financiera: https://www.juntamonetariafinanciera.gob.ec/wp-content/uploads/downloads/2019/08/Res.No.-534-2019-F.pdf

Cedeño, J., Benavides, A., Domo, I., Peña, H., \& Peña, J. (febrero de 2019). Beneficios del uso del dinero electrónico en el nivel de ventas de las Pymes. Obtenido de Revista Ibérica de Sistemas y Tecnologías de Información: https://search.proquest.co

González, J. (2019). La Digitalización del dinero. Obtenido de Boletín Oficial del Estado Español: https://www.boe.es/biblioteca_juridica/anuarios_derecho/abrir_pdf.php?id=ANU-M-2019- 
10025700278_ANALES_DE_LA_REAL_ACADEMIA_DE_CIENCIAS_MORALES_Y_POL\%C 3\%8DTICAS_La_digitalizaci\%C3\%B3n_del_dinero

INEC. (2020). Metodología de carga del Servicio de Rentas Internas. Obtenido de Instituo Nacional de Estadística y Censos: http://anda.inec.gob.ec/anda/index.php/catalog/513/download/7401

López, A., \& Baquerizo, L. (1 de septiembre de 2018). Manejo del dinero electrónico en Ecuador y su incidencia del traspaso a la banca privada. Obtenido de Revista Polo del Conocimiento: https://polodelconocimiento.com/ojs/index

Luque, A., Morales, T., \& Machado, L. (2019). Dinero electrónico, desconocimiento y resistencias: el caso de Ambato y Machala en Ecuador. Obtenido de Revista Ciencias Pedagógicas e Innovación UPSE: http://dx.doi.org/10.26423/rcpi.v5i3.210

Machuca, P., Iglesias, L., Ruperti, J., \& Moreira, M. (29 de enero de 2018). La moneda electrónica en el sistema financiero ecuatoriano. Obtenido de Revista Científica Multidisciplinaria Mikarimin: http://45.238.216.13/ojs/index.php/ mikarimin/article/vie

nippon.com. (4 de octubre de 2019). Japón sigue dependiendo del dinero en efectivo. Obtenido de nippon.com: https://www.nippon.com/es/japan-data/h00417/

Paredes, P. (julio de 2016). Evasión Tributaria vs Mecanismos implementados por la Administración Pública. Obtenido de Retos Revista de Ciencias de Administración y Economía: https://retos.ups.edu.ec/index.php/retos/article/view/12.2016.04

Pilay, F. (11 de junio de 2018). Pago desde mi Celular con dinero electrónico. Obtenido de Revista Científica Hallazgos: https://revistas.pucese.edu.ec/hallazgos21/article/view/247

Pineda, J. (noviembre de 2019). El rol del dinero electrónico en el marco de la estrategia nacional de inclusión financiera en el Perú. Obtenido de Pontificia Universidad Católica del Perú: http://tesis.pucp.edu.pe/repositorio/bitstream/handle/20.500.12404/15654/ABARCA_PAUCAR EDGAR_ENRIQUE.pdf?sequence $=1$ \&isAllowed $=y$

Quiñonez, E., Durán, G., Vera, P., \& Brito, O. (25 de octubre de 2019). El dinero electrónico como medio de pago para la inclusión tributaria en Ecuador. Obtenido de Revista Innova Research Journal: https://doi.org/10.33890/innova.v1.n10.2016.38

Reinoso, C., González, R., \& Gallegos, C. (junio de 2018). El dinero electrónico y su participación en la economía mundial y el cooperativismo. Obtenido de Revista Observatorio de la Economía Latinoamericana: https://www.eumed.net/rev/oel/2018/06/dine

Rivera, A., Hablich, F., \& Berni, L. (2018). Dinero electrónico: Beneficios tributarios. Obtenido de Revista Global de Negocios: https://poseidon01.ssrn.com/delivery.php?ID=05611211800009300212510111200209012211 808206303706102808112212007311

Roa, M., García, N., Frías, A., \& Correa, L. (octubre de 2017). Panorama del dinero móvil en América Latina y el Caribe. Obtenido de Centro de Estudios Monetarios Latinoamericanos CEMLA: https://www.cemla.org/PDF/otros/2017-06-panorama-del-dinero-movil.pdf

Rubio, J., Pérez, B., Acosta, D., \& Arroyo, J. (23 de marzo de 2021). Preferencias en el uso de pagos electrónicos en el Ecuador. Obtenido de Banco Central del Ecuador: https://estudioseconomicos.bce.fin.ec/index.php/RevistaCE/article/view/292/207 
Salas, E. (1 de julio de 2020). El dinero electrónico, una modalidad de pago moderna y segura. Obtenido de Universidad Técnica Particular de Loja: https://dialoguemos.ec/2020/07/eldinero-electronico-una-modalidad-de-pago-moderna-y-segura/

SRI. (2020). Régimen Impositivo Simplificado Ecuatoriano. Obtenido de Servicio de Rentas Internas: https://www.sri.gob.ec/web/guest/regimen-impositivo-simplificado-rise

SRI. (2020). Registro Único de Contribuyentes. Obtenido de Servicio de Rentas Internas: https://www.sri.gob.ec/web/guest/RUC

Valencia, F. (marzo de 2019). Sistema de Dinero Electrónico, un medio de pago al alcance de todos. Obtenido de Centro de Estudios Monetarios Latinoamericanos CEMLA: https://www.cemla.org/PDF/boletin/PUB_BOL_LX04-02.pdf

Villaroel, V., Ortíz, H., \& Proaño, T. (2018). El dinero electrónico y su incidencia en el sistema cooperativo de la ciudad de Ambato - Provincia de Tungurahua. Obtenido de Pontificia Universidad Católica del Ecuador: https://repositorio.pucesa.edu.ec/bitstream/123456789/2022/1/Dinero\%20Electr\%C3\%B3nico .pdf

Villegas, F., Loor, B., Montero, C., \& Fabiani, B. (diciembre de 2019). Análisis de aceptación del Sistema de Dinero Electrónico (SDE) frente a los riesgos del mercado económico actual del cantón Milagro, Ecuador. Obtenido de Revista Ciencia UNEMI: http://cienciaunemi.unemi.edu.ec/index.php/cienciaunemi/article/view/375/311 